\title{
Characteristics of Strange Hadron Production in Some High Energy Collisions and the Role of Power Laws
}

\author{
Sunil Kumar Biswas ${ }^{1}$, Goutam Sau ${ }^{2}$, Amar Chandra Das Ghosh ${ }^{3}$, Subrata Bhattacharyya ${ }^{4 *}$ \\ ${ }^{1}$ West Kodalia Adarsha Siksha Sadan, New Barrackpore, Kolkata, India \\ ${ }^{2}$ Beramara RamChandrapur High School, Kolkata, India \\ ${ }^{3}$ Department of Microbiology, Surendranath College, Kolkata, India \\ ${ }^{4}$ Physics and Applied Mathematics Unit (PAMU), Indian Statistical Institute, Kolkata, India \\ Email: \{sunil_biswas2004, sau_goutam\}@yahoo.com,dasghosh@yahoo.co.in, \\ *bsubrata@www.isical.ac.in
}

Received November 8, 2011; revised December 28, 2011; accepted January 10, 2012

\begin{abstract}
Studies on "strange" particle production have always occupied a very important space in the domain of Particle Physics. This was and is so, just because of some conjectures about specially abundant or excess production of "strange" particles, at certain stages and under certain conditions arising out of what goes by the name of "Standard" model in Particle Physics. With the help of Hagedornian power laws we have attempted to understand and interpret here the nature of the $p_{T}$-spectra for the strange particle production in a few high energy nuclear collisions, some interesting ratio-behaviors and the characteristics of the nuclear modification factors that are measured in laboratory experiments. After obtaining and analysing the final results we do not confront any peculiarities or oddities or extraneous excesses in the properties of the relevant observables with no left-over problems or puzzles. The model(s) used by us work(s) quite well for explaining the measured data.
\end{abstract}

Keywords: Hadron-Nucleus Collisions; Inclusive Production; Scaling Phenomena; Power Laws

\section{Introduction}

Studying the nature of particle production in protonproton collisions is important and interesting in itself, as it might shed light on the basic mechanism for production of particles. Besides, it could also serve as a necessary benchmark for the physics developments in ultra-relativistic heavy ion collisions [1,2]. This is specially important at the large hadron collider (LHC) where the heavy ion programme had started by November 2010 delivering some preliminary results on some aspects of strange hadrons produced as the final product in high energy nuclear collisions and these strange secondaries are supposed to provide valuable insights into the properties of the "controversial" system newly formed. One of the main motivation for measuring strange particles in heavy nucleus-nucleus reactions at LHC is the expectation that their production-rates for participating nucleon should be enhanced with respect to basic nucleon-nucleon interactions. Strangeness enhancement has consistently been proposed as one of the strong diagnostics for a Quark Gluon Plasma (QGP) state $[3,4]$. The enhancement factor (E) is defined as rapidity-density

"Corresponding author. of multiplicity (yield) par mean number of nucleon participants $\left[\left\langle N_{p a r t}\right\rangle\right]$ in heavy ion collisions, divided by the respective value in $p+p$ collisions. The requisite information about $\left\langle N_{\text {part }}\right\rangle$ etc are to be obtained from Abelev et al. [5,6].

As the strange hadrons are not at all present in the initial system (A), the question rises very sharply: how do they make their appearance in the final products. So there must be some specific reflections on the constituent pictures of the particles and specifically the nucleons. Besides, enhancement of strangeness productions was/is one of the powerful diagnostics for the formation of quark gluon plasma (QGP) in relativistic heavy ion collisions and the colliders (RHIC). The observations of an increase of strange baryon production relative to $p+p$ collision in SPS data, confirmed later at the RHIC studies, has brought excitement in this area.Besides, the increase of $p / \pi$ ratio (B) in such collisions in the non-strange domain had its parallel in the strangeness sector with the observation of slow rise of the $\Lambda / k^{0}$ values.

The organization of this work is as follows. In Section 2 we give an outline of the model to be applied. In the next section (Section 3) we deliver the results by figures and tables with a short discussion on the results obtained. 
In the last chapter, we precisely point out the conclusions to be arrived at.

\section{The Background in Some Detail and the Working Formulae}

With gradual attainments of larger transverse momenta $\left(p_{T}\right)$ of the secondaries in high- $p_{T}$ (hard) interactions, the problems of deviations from exponential nature of fits on invariant spectra began to crop up steadily. Gazdzicki and Gorenstein [7] observed rightly that for $p_{T}>2 \mathrm{GeV} / \mathrm{c}$, the data sharply deviate from the exponential nature, for which Darriulat[8] proposed a power law distribution of certain forms for both $p_{T}$ spectra and particle multiplicity. Indeed, for both $p_{T}$ spectra and multiplicity such power law forms have become now the most dominant tools in dealing with the transverse momentum spectra of all hadrons. Gazdzicki and Gorenstein showed that the normalised multiplicities and $\left(m_{T} \sim p_{T}\right)$ spectra of neutral mesons obey the $m_{T}$-scaling which has had an approximately power law structure of the form $\sim\left(m_{T}\right)^{n}$, where $m_{T}$ is called transverse mass and is defined by $m_{T}=\sqrt{p_{T}^{2}+m^{2}}$. This scaling behaviour was analogous to that expected in statistical mechanics, the parameter $\mathrm{n}$ plays the role of temperature and any normalization constant to be used resembles the system volume.Thus the basic modification of the statistical approach needed to reproduce the experimental results on some hadron production process in $p(\bar{p})+p$ interaction in the large $m_{T} \equiv p_{T}$ domain is to change the shape of the distribution functions $\exp \left(-\frac{E^{*}}{T}\right)$ had to be altered to the power law form as given by $\left(\frac{E^{*}}{\Lambda}\right)^{-n}$ with some changed parameters, viz, a scale parameter $\Lambda$ and an exponent $n$, both are assumed to be common for all hadrons.

Let us now dwell, in brief, on the clues to the possible origin of power laws. One of the basic features of the hadronuclear collisions is: irrespective of the initial state,agitations caused by the impinging projectile (be it a parton or particle/nucleon) generate system effects of producing avalanches of new kind of partons (called gluons) which form an open dissipative system.And these production processes are not at all gradual; rather they are very sudden,drastic and complex. And such complex properties and processes in nature do generally subscribe to the power-law behaviours.In the recent times, it is being propounded consistently that the power law behaviours put into use here are "manifestations of the dynamics of complex systems whose striking feature is of showing universal laws characterized by exponents in scale invariant distributions that happen to be basically independent of the details in the microscopic dynamics" [9]. The avalanches caused by production of excessive number of some new variety of parton called 'gluons' (the process called "gluonisation") give rise to the jettiness of particle production and of cascadisation of the particle production processes leading to the fractality as is shown by Sarcevic [10]. These cascades are self-organizing, self-similar and do just have the fractal behaviour. Driven by the physical impacts of these well-established factors, in the high energy collision processes do crop up the several power-laws.And how such power laws do evolve from exponential origins or roots is now-a-days being taken care of by the induction of Tsallis entropy [11] and a generalisation of GibbsBoltzmann statistics for long-range and multifractal processes.

In what follows we are going to choose a specific form of power law which was previously applied by us in the case of hadron-nucleus collisions. With a view to accommodating some observed facts for strange particle production, it is tempting to try to fit the whole distribution for the inclusive $p_{T}$-spectra with one single expression in the form of power law as was done by G. Arnison et al. [12] and Hagedorn [13].

$$
E \frac{\mathrm{d}^{3} \sigma}{\mathrm{d} p^{3}}=\text { const. } \frac{d(\mathrm{~d} N / \mathrm{d} y)}{2 \pi p_{T} d p_{T}}=A\left(\frac{q}{p_{T}+q}\right)^{n}
$$

where the letters and expressions have their contextual significance.This parametrization describe the data well over the entire range of $p_{T}$.

Indeed for $p_{T} \rightarrow 0, \infty$ we have,

$$
\begin{aligned}
& \left(\frac{q}{p_{T}+q}\right)^{n} \approx\left(1-\frac{n}{q} p_{T}\right)
\end{aligned}
$$

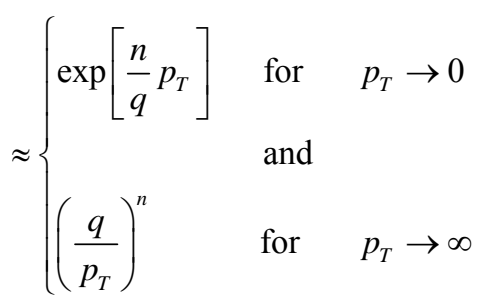

Thus along with impressive fit, which now includes the large $p_{T}$ domain, the estimate of $\left\langle p_{T}\right\rangle$ assumes with the help of expression (1):

$$
\left\langle p_{T}\right\rangle=\frac{\int q /\left(p_{T}+q\right)^{n} p_{T}^{2} \mathrm{~d} p_{T}}{\int q /\left(p_{T}+q\right)^{n} p_{T} \mathrm{~d} p_{T}}=\frac{2 q}{n-3}
$$

So, in clearer terms, let us put the final working formulae here as follows with substitution of $p_{T}$ (transverse momentum) as $x$ in the power-law model $[14,15]$ respectively 


$$
f(x)=A(1+x / q)^{-n}
$$

There is yet another very important observable called nuclear modification factor (NMF), denoted here by $R_{C P}$, which for production of any hadron is defined by [16]

$$
R_{C P}\left(p_{T}\right)=\frac{\left[\left(d^{2} N /\left(2 \pi \mathrm{d} p_{T} \mathrm{~d} p_{T} \mathrm{~d} y\right)\right)_{c} / N_{\text {bin }}\right]^{\text {Cenrral }}}{\left[\left(d^{2} N /\left(2 \pi \mathrm{d} p_{T} \mathrm{~d} p_{T} \mathrm{~d} y\right)\right)_{c} / N_{\text {bin }}\right]^{\text {Peripheral }}}
$$

\section{Results}

In obtaining the results presented here, no serious statistical calculational procedure was adopted. The graphs are drawn more as fitological-cum-phenomenological exercises with mainly statistical errors in considerations. The experimental data do not provide, in the most cases, any systematic errors. Data points for the heavy, high strangeness-valued particles are too scarce; for which the

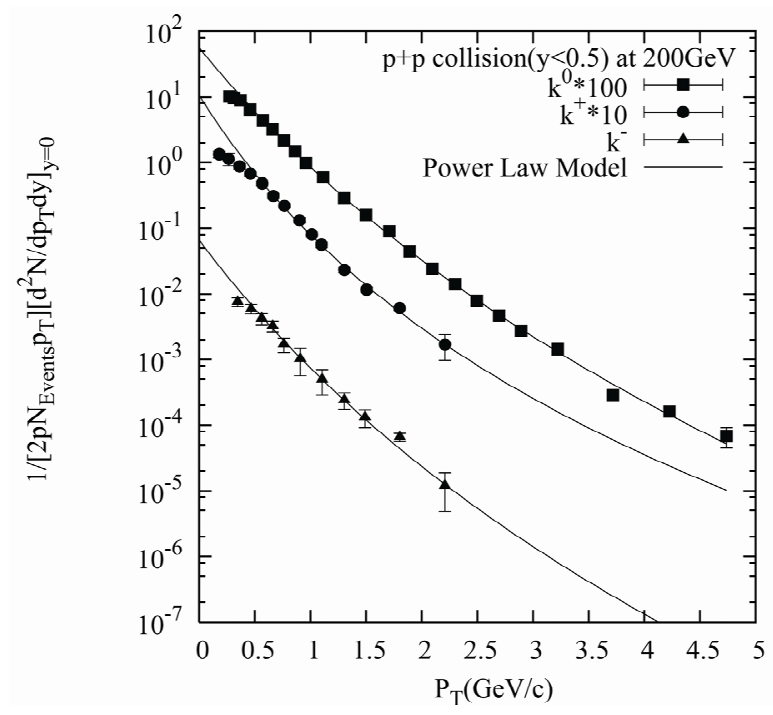

(a) number of degrees of freedom is too limited for many cases. The quality of fits to the data indicated in the tables by $\chi^{2} / n d f$ terms in the columns is attempted to be kept at a modestly satisfactory value (tending as nearly as possible to unity). And the figures are drawn by Wgnuplot, wherein there are some inbuilt statistical procedures and techniques.

Quite observably, the results are depicted here in graphical plots. And the used values of the corresponding parameters for fits are shown in separate tables. The Figures 1(a) and (b) show the production of secondaries $k^{0}, k^{+}, k^{-}, \Lambda$ and $\bar{\Lambda}$ in proton-proton collisions at $\sqrt{s}=200 \mathrm{GeV}$ at the rapidity $y<0.5$ (Table 1). The figures in Figures 2(a) and (b) depict the results for the $\Xi^{-}, \bar{\Xi}^{+}, \Lambda, \bar{\Lambda}$ particle production for the same collision at the same energy (Table 2). The cases of $k^{+}$and $k^{-}$ production in gold-gold reaction at the same energy and at different centralities are reproduced by power laws in Figures 3(a) and (b) (Table 3). In Figures 4(a) and (b)

Figure 1. Transverse momentum spectra for production of keon $\left(K^{0}, K^{+}, K^{-}\right)$, lamda $(\Lambda)$, lamdabar $(\bar{\Lambda})$, cascade minus $\left(\Xi^{-}\right)$, cascade plus bar ( $\Xi^{+}$bar $)$particles in pp collisions at $\sqrt{s}=200 \mathrm{GeV}$. The experimental data are taken from Ref. [5]. The solid curves are fits for power-law model.

Table 1. Numerical values of the fit parameters of power law equation for keon and lamda production in p-p collisions at $\sqrt{s_{N N}}=200 \mathrm{GeV}, p_{T}=0$ to $5 \mathrm{GeV} / \mathrm{c}$.

\begin{tabular}{ccccc}
\hline Sesondaries & $A$ & $q$ & $n$ & $\frac{\chi^{2}}{n d f}$ \\
\hline$K_{s}^{0}$ & $0.563 \pm 0.023$ & $3.108 \pm 0.025$ & $15.005 \pm 0.032$ & $20.707 / 17$ \\
$K^{+}$ & $1.067 \pm 0.032$ & $1.581 \pm 0.055$ & $10.000 \pm 0.209$ & $10.943 / 9$ \\
$K^{-}$ & $0.066 \pm 0.008$ & $2.895 \pm 0.026$ & $15.116 \pm 0.405$ & $0.584 / 6$ \\
$\Lambda$ & $0.273 \pm 0.036$ & $3.092 \pm 0.068$ & $15.007 \pm 0.074$ & $18.393 / 15$ \\
$\Lambda$ bar & $0.029 \pm 0.001$ & $3.010 \pm 0.068$ & $15.016 \pm 0.068$ & $15.673 / 15$ \\
\hline
\end{tabular}




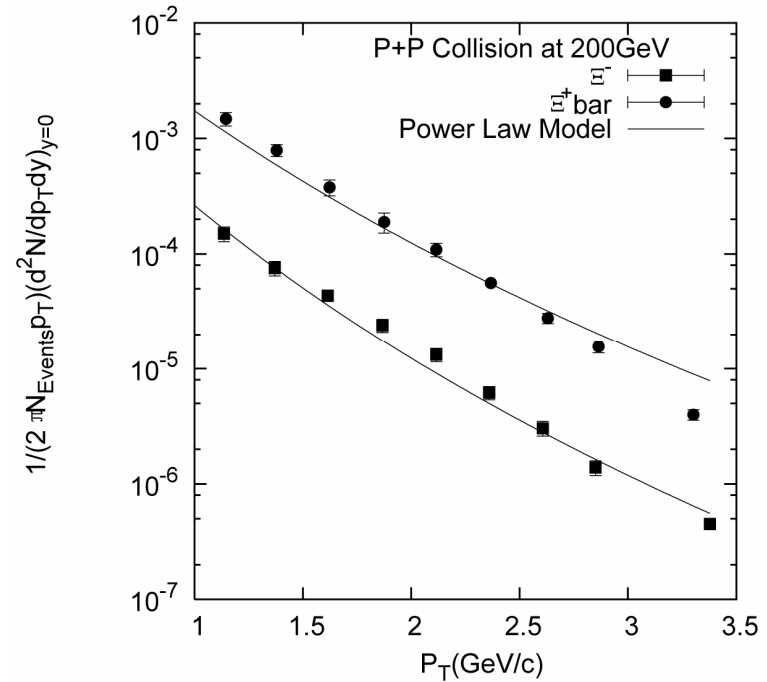

(a)

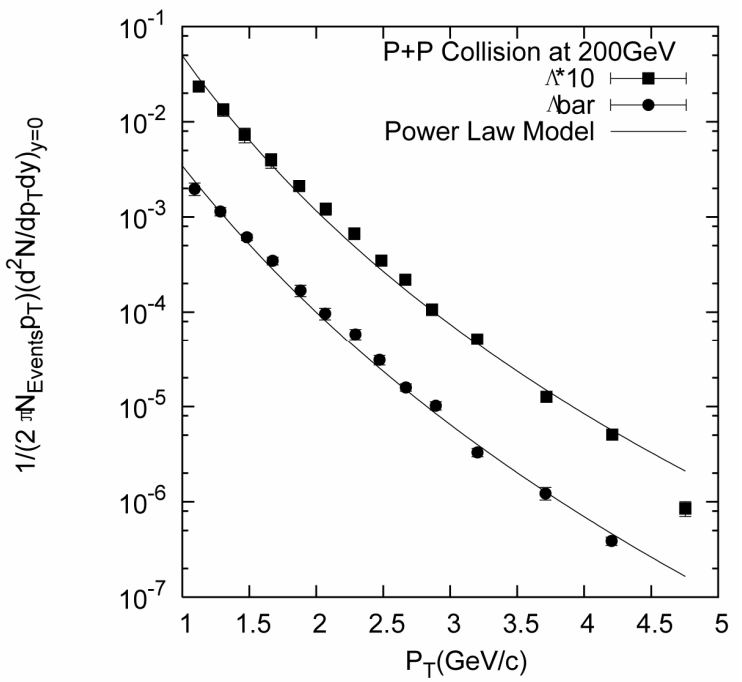

(b)

Figure 2. Transverse momentum spectra for production of cascade $\left(\Xi^{-}, \Xi^{+}\right.$bar $)$, lamda and lamdabar particles in pp collisions at $\sqrt{s}=200 \mathrm{GeV}$. The experimental data are taken from Ref. [17]. The solid curves are fits for power-law model.

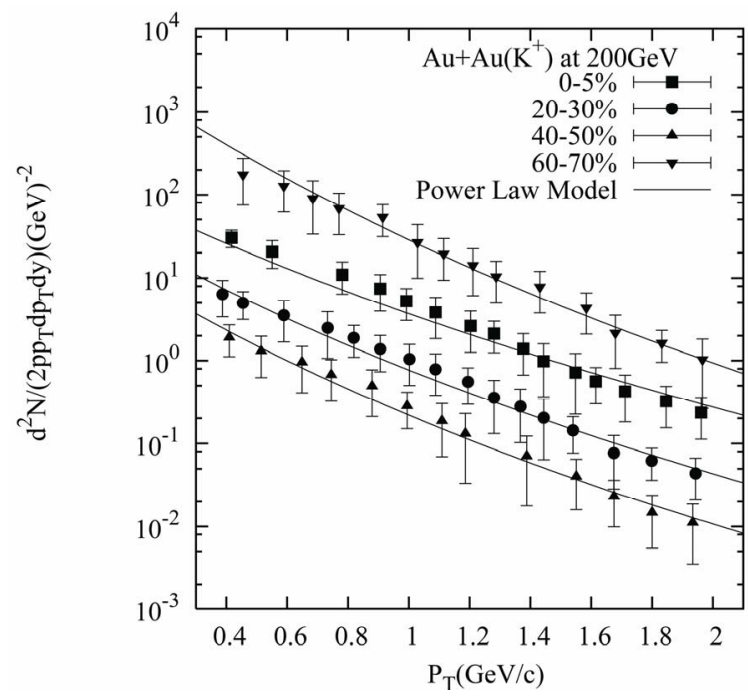

(a)

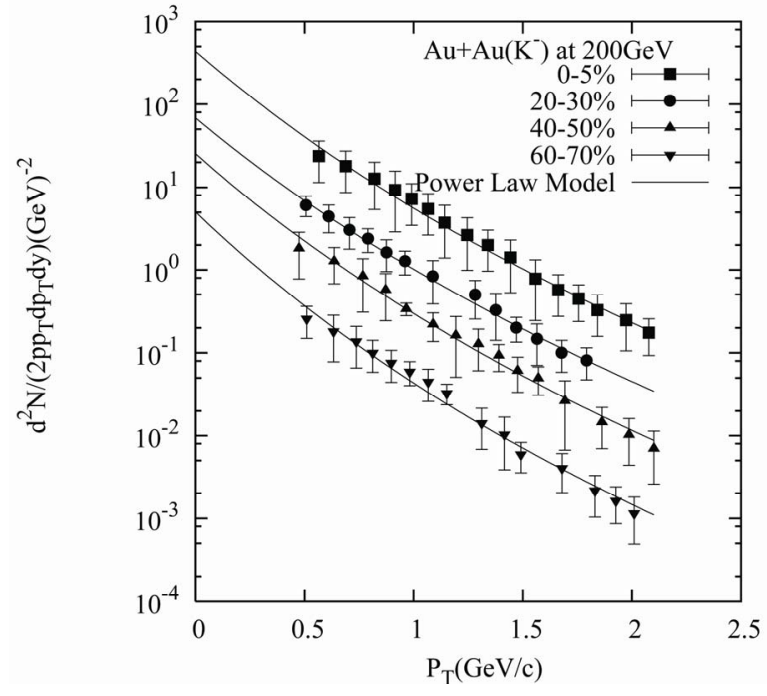

(b)

Figure 3. Transverse momentum spectra for production of kaon $\left(K^{+}, K^{-}\right)$at different centrality at $\sqrt{s}=200 \mathrm{GeV}$ in $\mathrm{Au}-\mathrm{Au}$ collisions. The experimental data are taken from Ref. [18]. The solid curves are fits for power-law model.

Table 2. Numerical values of the fit parameters of power law equation for lamda and cascade particle production in p-p collisions at $\sqrt{s_{N N}}=200 \mathrm{GeV}, p_{T}=0$ to $5 \mathrm{GeV} / \mathrm{c}$.

\begin{tabular}{ccccc}
\hline Sesondaries & $A$ & $q$ & $n$ & $\frac{\chi^{2}}{n d f}$ \\
\hline$\Lambda$ & $1.848 \pm 0.094$ & $1.306 \pm 0.061$ & $10.417 \pm 0.244$ & $26.883 / 12$ \\
$\Lambda b a r$ & $0.541 \pm 0.026$ & $1.910 \pm 0.068$ & $12.005 \pm 0.061$ & $36.675 / 13$ \\
$\Xi^{-}$ & $0.022 \pm 0.001$ & $1.804 \pm 0.023$ & $10.033 \pm 0.119$ & $21.4 / 8$ \\
$\Xi^{+}$bar & $0.063 \pm 0.002$ & $2.301 \pm 0.015$ & $9.951 \pm 0.466$ & $24.246 / 9$ \\
\hline
\end{tabular}




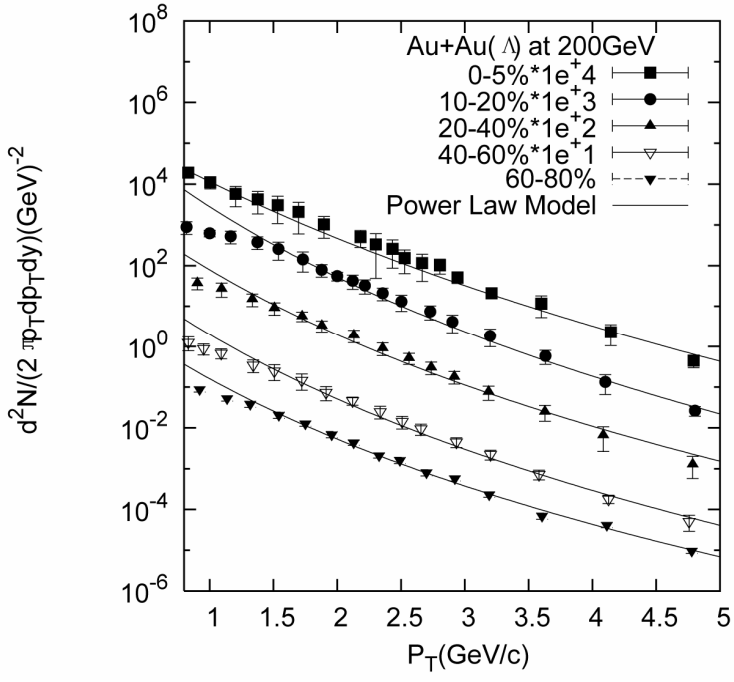

(a)

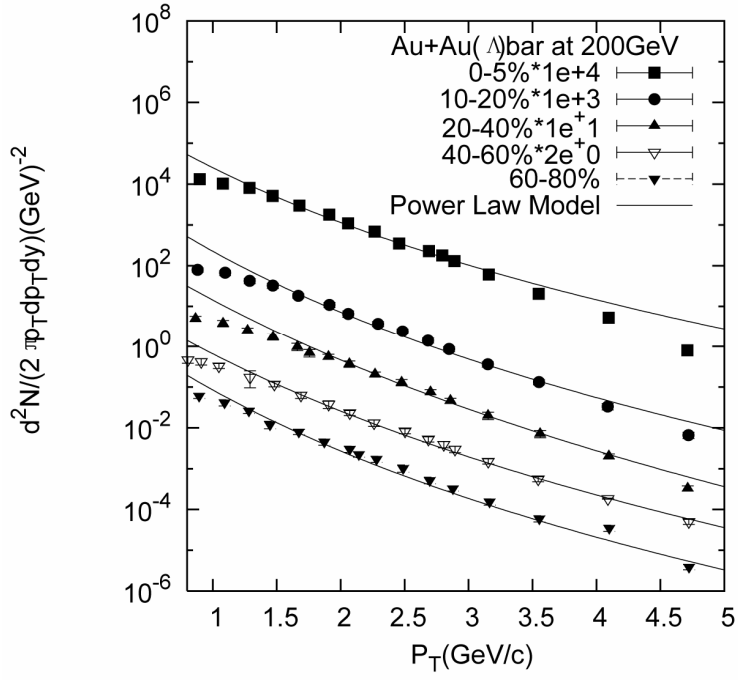

(b)

Figure 4. Transverse momentum spectra for production of lamda $(\Lambda)$ and lamda bar $(\bar{\Lambda})$ particles at different centrality in Au-Au collisions. The experimental data are taken from Ref. [18] The solid curves are fits for power-law model.

Table 3. Numerical values of the fit parameters of Power Law equation for keon production $\left(k^{+}, k^{-}\right)$in Au-Au collisions at $\sqrt{s_{N N}}=200 \mathrm{GeV}$ at different Centrality, $p_{T}=0$ to $2 \mathrm{GeV} / \mathrm{c}$.

\begin{tabular}{|c|c|c|c|c|c|}
\hline Secondaries & Centrality $(\%)$ & $A$ & $q$ & $n$ & $\frac{\chi^{2}}{n d f}$ \\
\hline \multirow{3}{*}{$k^{+}$} & $0-5 \%$ & $120.114 \pm 8.341$ & $2.508 \pm 0.057$ & $10.376 \pm 0.172$ & $4.555 / 14$ \\
\hline & $20 \%-30 \%$ & $47.371 \pm 2.28$ & $1.998 \pm 0.029$ & $9.989 \pm 0.112$ & $2.477 / 15$ \\
\hline & $40 \%-50 \%$ & $16.727 \pm 2.703$ & $1.843 \pm 0.082$ & $10.006 \pm 0.111$ & $1.294 / 12$ \\
\hline \multirow{4}{*}{$k^{-}$} & $0-5 \%$ & $435.675 \pm 15.85$ & $2.286 \pm 0.076$ & $12.00 \pm 0.264$ & $1.032 / 15$ \\
\hline & $20 \%-30 \%$ & $68.462 \pm 6.505$ & $2.379 \pm 0.061$ & $12.003 \pm 0.07$ & $0.246 / 12$ \\
\hline & $40 \%-50 \%$ & $25.454 \pm 0.901$ & $2.239 \pm 0.084$ & $12.052 \pm 0.297$ & $1.566 / 14$ \\
\hline & $60 \%-70 \%$ & $5.058 \pm 0.283$ & $2.016 \pm 0.101$ & $11.819 \pm 0.382$ & $3.356 / 14$ \\
\hline
\end{tabular}

the production of $\Lambda$ and $\bar{\Lambda}$ are shown in the same collision at the same energy(Table 4). In Figures 5(a), (b) and (c) the production of cascade, cascade-bar and omega particles production are shown at different centralities and at the same energy (Table 5). The cases of production of neutral kaons and lamda particles in Copper-Copper collision at $\sqrt{s}=200 \mathrm{GeV}$ are plotted in Figures 6(a) and (b) with reckoning of the parameters presented in Tables 6 and $\mathbf{7 .}$

In Table 8 the values of average transverse momenta $\left(\left\langle p_{T}\right\rangle\right.$ ) for different produced secondaries in protonproton and gold-gold collisions have been computed. All these values tally with the similar ranges arrived at by experimental measurements. This helps us to obtain for us a consistency check-up of the parameter values used for getting fits to the data on $\left\langle p_{T}\right\rangle$-spectra. In Figures 7(a) and (b) we see, the lamda-bar to lamda and cascademinus to cascade-plus particle production cross-section ratio as a function of transverse momentum respectively and the ratio gradually fall off with increasing values of $p_{T}$. In Figures 8(a) and (b) the nuclear modification factors $\left(R_{C P}\right)$ are plotted against transverse momentum for the production of neutral meson and lamda particles in copper-copper collision. With the increasing $p_{T}$, the $R_{C P}$-values fall off. In addition, the data show the $R_{C P}$ for baryons exhibits a lower fall-off compared with that of mesons in intermediate transverse momentum region. The experimental data show that the baryonmeson difference of $R_{C P}$ disappears at higher $p_{T}$.

The data on production of strange particles described 
Table 4. Numerical values of the fit parameters in Power Law form for ( $\Lambda$ and $\bar{\Lambda}$ ) production in Au-Au collisions at $\sqrt{s_{N N}}=200 \mathrm{GeV}$ at $p_{T}=1$ to $5 \mathrm{GeV} / \mathrm{c}$ ranges and for various Centrality-values as given below.

\begin{tabular}{|c|c|c|c|c|c|}
\hline Secondaries & Centrality (\%) & $A$ & $q$ & $n$ & $\frac{\chi^{2}}{n d f}$ \\
\hline \multirow{4}{*}{$\Lambda$} & $0-5 \%$ & $63.570 \pm 4.841$ & $3.542 \pm 0.105$ & $16.124 \pm 0.140$ & $10.064 / 18$ \\
\hline & $10 \%-20 \%$ & $772.323 \pm 48.46$ & $2.000 \pm 0.087$ & $13.870 \pm 0.259$ & $5.778 / 14$ \\
\hline & $20 \%-40 \%$ & $141.42 \pm 11.33$ & $2.002 \pm 0.023$ & $12.815 \pm 0.099$ & $7.512 / 12$ \\
\hline & $60 \%-80 \%$ & $19.811 \pm 0.968$ & $2.000 \pm 0.014$ & $11.869 \pm 0.235$ & $14.913 / 12$ \\
\hline \multirow{4}{*}{$\Lambda b a r$} & $0-5 \%$ & $198.233 \pm 0.269$ & $2.000 \pm 0.014$ & $10.765 \pm 0.269$ & $7.588 / 10$ \\
\hline & $10 \%-20 \%$ & $29.161 \pm 1.727$ & $2.002 \pm 0.121$ & $12.003 \pm 0.072$ & $17.298 / 10$ \\
\hline & $20 \%-40 \%$ & $122.194 \pm 4.406$ & $2.899 \pm 0.014$ & $15.001 \pm 0.054$ & $3.589 / 8$ \\
\hline & $60 \%-80 \%$ & $10.994 \pm 0.788$ & $2.001 \pm 0.022$ & $11.995 \pm 0.088$ & $31.900 / 13$ \\
\hline
\end{tabular}

Table 5. Numerical values of the fit parameters in Power Law equation for cascade-minus ( $\Xi^{-}$), cascade-plus bar ( $\Xi^{+}$bar ) and $\Omega^{-}+\Omega^{+}$particle production in Au-Au collisions at $\sqrt{s_{N N}}=200 \mathrm{GeV}$ at different $p_{T}$-values $=1$ to $5 \mathrm{GeV} / \mathrm{c}$ for various Centrality values.

\begin{tabular}{|c|c|c|c|c|c|}
\hline Secondaries & Centrality (\%) & $A$ & $q$ & $n$ & $\frac{\chi^{2}}{n d f}$ \\
\hline \multirow{5}{*}{$\Xi^{-}$} & $0-5 \%$ & $213.632 \pm 16.06$ & $1.344 \pm 0.015$ & $10.042 \pm 0.271$ & $14.593 / 8$ \\
\hline & $10 \%-20 \%$ & $1019.24 \pm 81.73$ & $1.001 \pm 0.010$ & $10.017 \pm 0.058$ & $20.800 / 6$ \\
\hline & $20 \%-40 \%$ & $2978.87 \pm 155.4$ & $1.000 \pm 0.006$ & $11.003 \pm 0.001$ & $6.138 / 6$ \\
\hline & $40 \%-60 \%$ & $16.505 \pm 0.288$ & $1.500 \pm 0.003$ & $10.019 \pm 0.055$ & $0.675 / 7$ \\
\hline & $60 \%-80 \%$ & $3.065 \pm 0.339$ & $1.503 \pm 0.027$ & $10.001 \pm 0.111$ & $16.968 / 5$ \\
\hline \multirow{5}{*}{$\Xi^{+} b a r$} & $0-5 \%$ & $1531.25 \pm 144$ & $1.001 \pm 0.012$ & $9.996 \pm 0.070$ & $18.845 / 7$ \\
\hline & $10 \%-20 \%$ & $5248.25 \pm 468.3$ & $0.801 \pm 0.008$ & $10.011 \pm 0.053$ & $22.498 / 6$ \\
\hline & $20 \%-40 \%$ & $735.176 \pm 31.68$ & $0.999 \pm 0.005$ & $9.996 \pm 0.031$ & $24.258 / 8$ \\
\hline & $40 \%-60 \%$ & $233.981 \pm 15.39$ & $0.999 \pm 0.008$ & $9.997 \pm 0.047$ & $16.173 / 7$ \\
\hline & $60 \%-80 \%$ & $2.993 \pm 0.188$ & $1.496 \pm 0.014$ & $10.006 \pm 0.057$ & $4.049 / 4$ \\
\hline \multirow{3}{*}{$\Omega^{-}+\Omega^{+}$} & $0-5 \%$ & $1.248 \pm 0.094$ & $3.018 \pm 0.055$ & $9.988 \pm 0.139$ & $2.073 / 3$ \\
\hline & $20 \%-40 \%$ & $2.970 \pm 0.229$ & $1.997 \pm 0.028$ & $9.978 \pm 0.100$ & $1.304 / 2$ \\
\hline & $40 \%-60 \%$ & $0.342 \pm 0.037$ & $2.001 \pm 0.043$ & $9.235 \pm 0.131$ & $4.755 / 3$ \\
\hline
\end{tabular}

here pertain, in the main, to the "hard" sector of high energy interactions. And it is well-known that Hagedornian power law forms which have their roots in the physics of quantum chromodynamics (QCD) describe hard particle production in a modestly successful manner for, at least, the light hadrons of which strange K-mesons constitute a part.But some of the strange particles have moderately high masses, for which our objective here was primarily to check whether this generalized power law form could address the issues of invariant $p_{T}$ spectra and some other related observables in a satisfactory manner for all the strange hadrons. And the outcome is: this study is strongly affirmative by all indications and yardsticks of actual performances.

The data on strange particles are in general quite sparse. The errors in measurements are also in most cases 


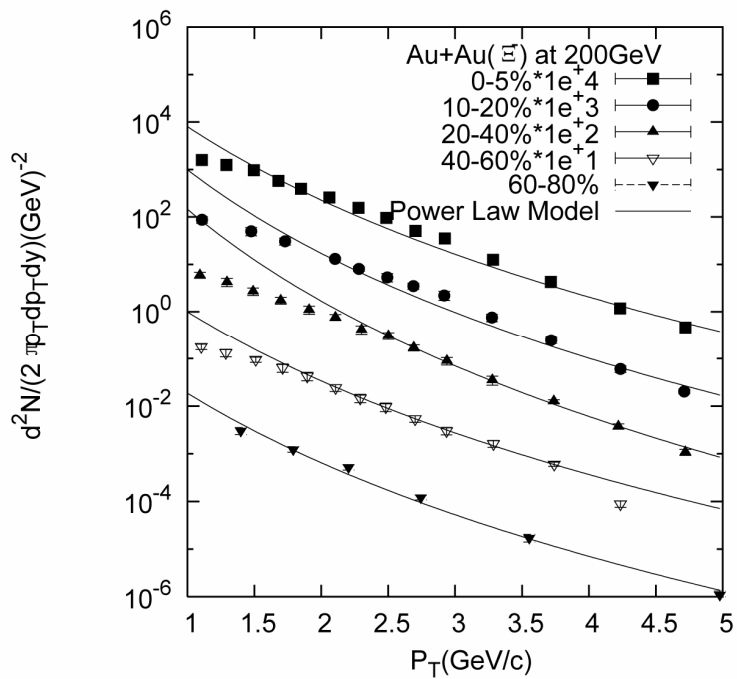

(a)

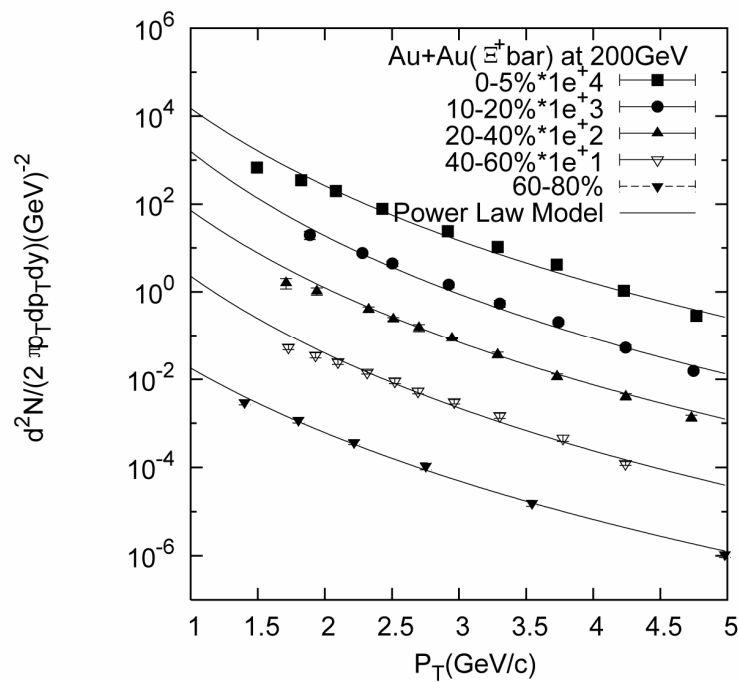

(b)

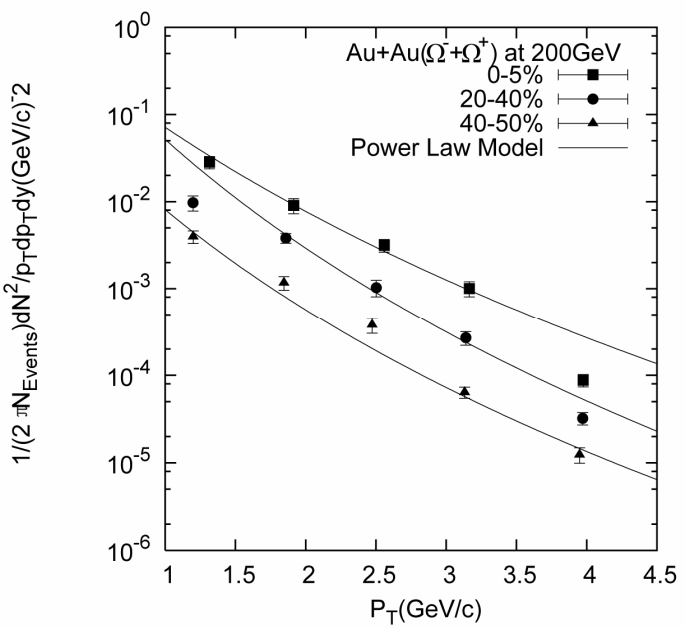

(c)

Figure 5. Transverse momentum spectra for production of cascade minus $\left(\Xi^{-}\right)$,cascade plus bar ( $\Xi^{+}$bar $)$and omega particles $\left(\Omega^{-}+\Omega^{+}\right)$at different centrality in Au-Au collisions at $\sqrt{s}=200 \mathrm{GeV}$. The experimental data are taken from Ref. [19]. The solid curves are fits for power-law model.

Table 6. Numerical values of the fit parameters of Power Law equation for keon production ( $\left.k_{s}^{0}\right)$ in $\mathrm{Cu}-\mathrm{Cu}$ collisions at $\sqrt{s_{N N}}=200 \mathrm{GeV}$ at different $p_{T}$-values $=1$ to $9 \mathrm{GeV} / \mathrm{c}$ and for several Centrality domains.

\begin{tabular}{cccccc}
\hline Secondaries & Centrality (\%) & A & $q$ & $n$ & $\frac{\chi^{2}}{n d f}$ \\
\hline & $0-10 \%$ & $112.079 \pm 4.169$ & $2.020 \pm 0.035$ & $12.229 \pm 0.094$ & $6.675 / 21$ \\
& $10 \%-20 \%$ & $67.284 \pm 2.154$ & $1.921 \pm 0.031$ & $11.712 \pm 0.477$ & $6.068 / 21$ \\
$k_{s}^{0}$ & $20 \%-30 \%$ & $24.860 \pm 0.568$ & $2.879 \pm 0.030$ & $14.155 \pm 0.262$ & $3.667 / 21$ \\
& $30 \%-40 \%$ & $17.099 \pm 0.286$ & $2.565 \pm 0.070$ & $13.181 \pm 0.0 .245$ & $6.230 / 21$ \\
& $40 \%-50 \%$ & $18.504 \pm 0.598$ & $1.985 \pm 0.036$ & $11.583 \pm 0.476$ & $7.738 / 21$ \\
& $50 \%-60 \%$ & $12.159 \pm 0.536$ & $2.000 \pm 0.006$ & $11.551 \pm 0.054$ & $7.842 / 21$ \\
\hline
\end{tabular}




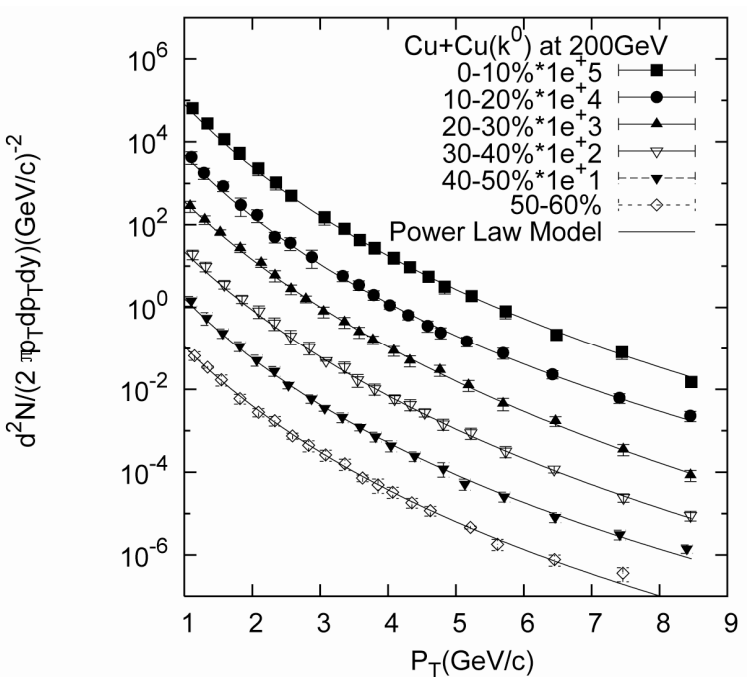

(a)

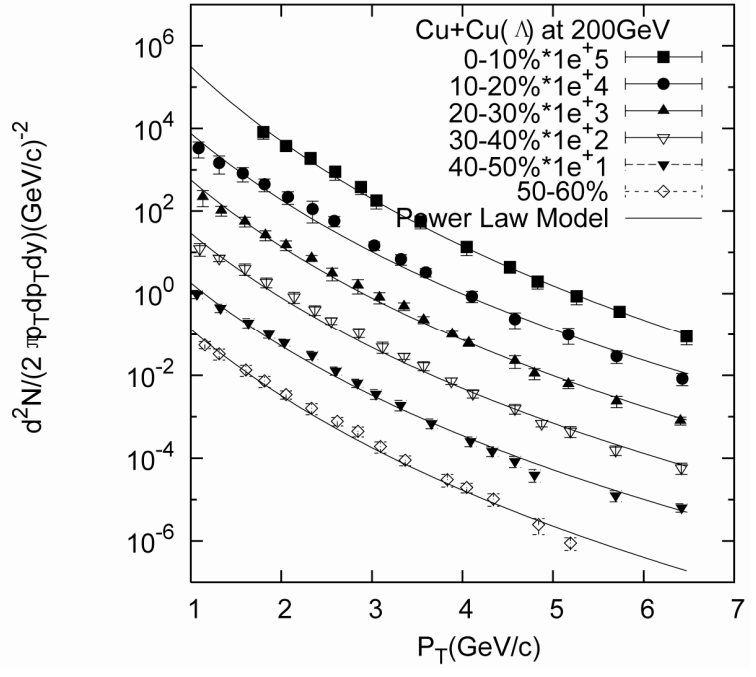

(b)

Figure 6. Transverse momentum spectra for production of neutral keons $\left(k_{s}^{0}\right)$ and lamda $(\Lambda)$ particles at different centrality in Au-Au collisions at $\sqrt{s}=200 \mathrm{GeV}$. The experimental data are taken from Ref. [18]. The solid curves are fits for power-law model.

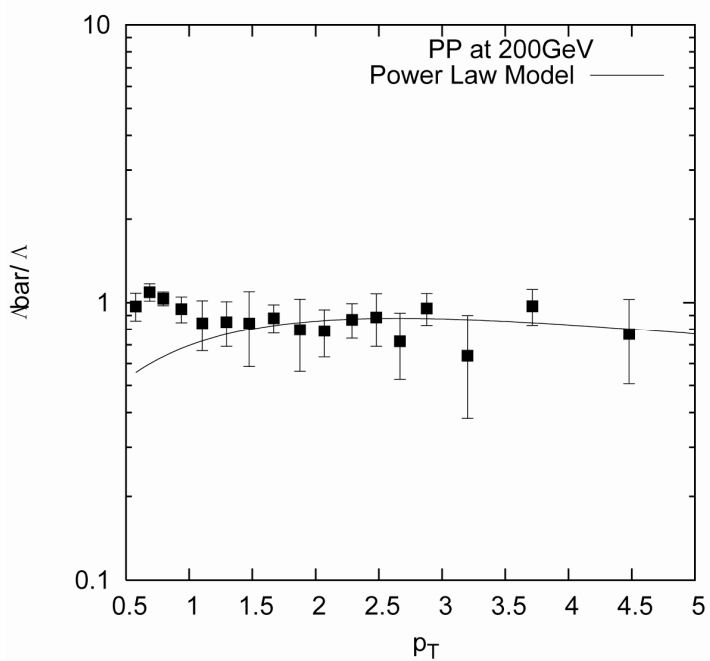

(a)

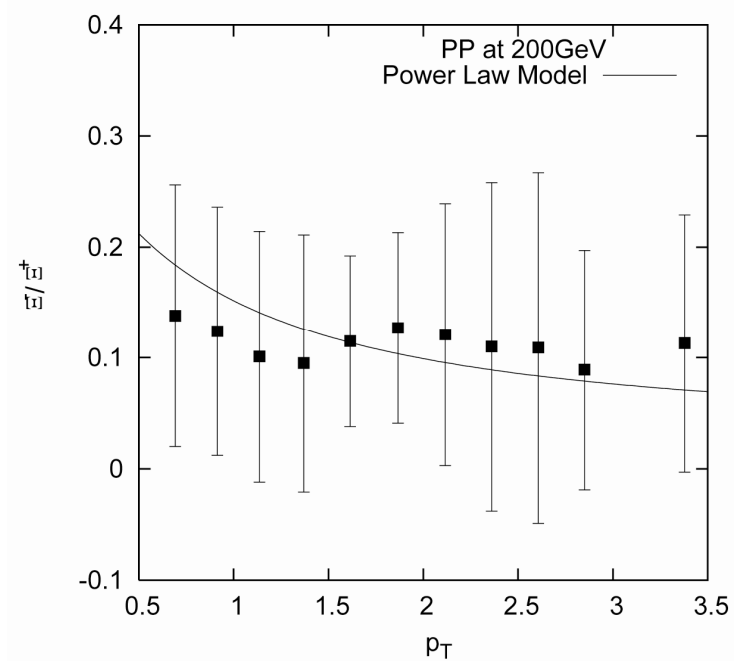

(b)

Figure 7. Transverse momentum-dependence spectra of $\Lambda \mathrm{bar} / \Lambda$ and $\Xi^{-} / \Xi^{+} \mathrm{bar}$ for pp collision at $\sqrt{\mathrm{s}}=200 \mathrm{GeV}$. The data type-points are taken from Ref. [5]. The solid curves or lines are drawn on the basis of Power Law Model.

Table 7. Numerical values of the fit parameters of Power Law equation for lamda particle $(\Lambda)$ production in $\mathrm{Cu}-\mathrm{Cu}$ collisions at $\sqrt{s_{N N}}=200 \mathrm{GeV}$ at different Centrality, $p_{T}=1$ to $7 \mathrm{GeV} / \mathrm{c}$.

\begin{tabular}{|c|c|c|c|c|c|}
\hline Secondaries & Centrality (\%) & $A$ & $q$ & $n$ & $\frac{\chi^{2}}{n d f}$ \\
\hline \multirow{6}{*}{$\Lambda$} & $0-10 \%$ & $1091.23 \pm 47.1$ & $2.000 \pm 0.009$ & $14.428 \pm 0.166$ & $2.635 / 12$ \\
\hline & $10 \%-20 \%$ & $139.73 \pm 12.91$ & $2.034 \pm 0.103$ & $13.033 \pm 0.275$ & $15.067 / 14$ \\
\hline & $20 \%-30 \%$ & $105.009 \pm 5.974$ & $2.024 \pm 0.073$ & $12.996 \pm 0.193$ & $16.425 / 17$ \\
\hline & $30 \%-40 \%$ & $46.450 \pm 1.523$ & $2.026 \pm 0.081$ & $12.629 \pm 0.216$ & $24.731 / 17$ \\
\hline & $40 \%-50 \%$ & $27.007 \pm 1.798$ & $2.001 \pm 0.018$ & $12.340 \pm 0.215$ & $16.803 / 15$ \\
\hline & $50 \%-60 \%$ & $24.819 \pm 2.077$ & $2.029 \pm 0.023$ & $13.042 \pm 0.097$ & $17.437 / 14$ \\
\hline
\end{tabular}




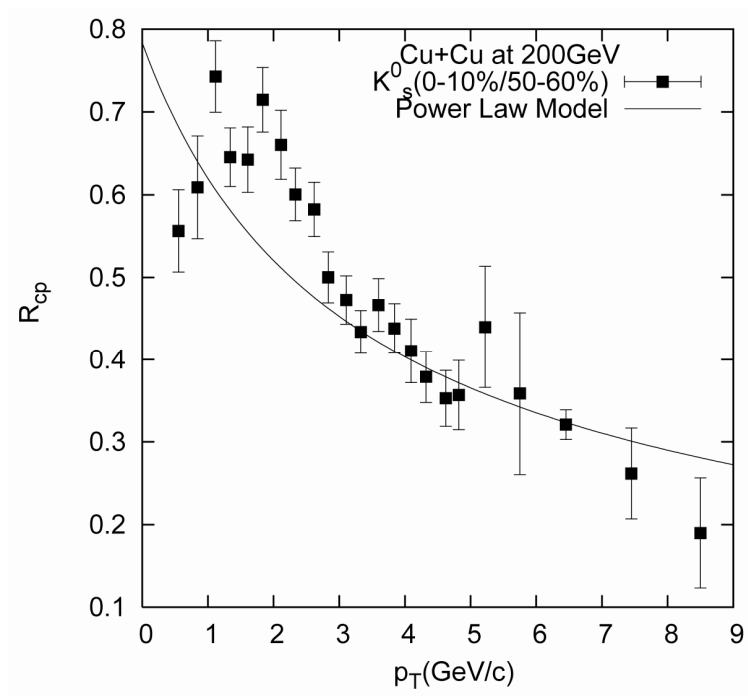

(a)

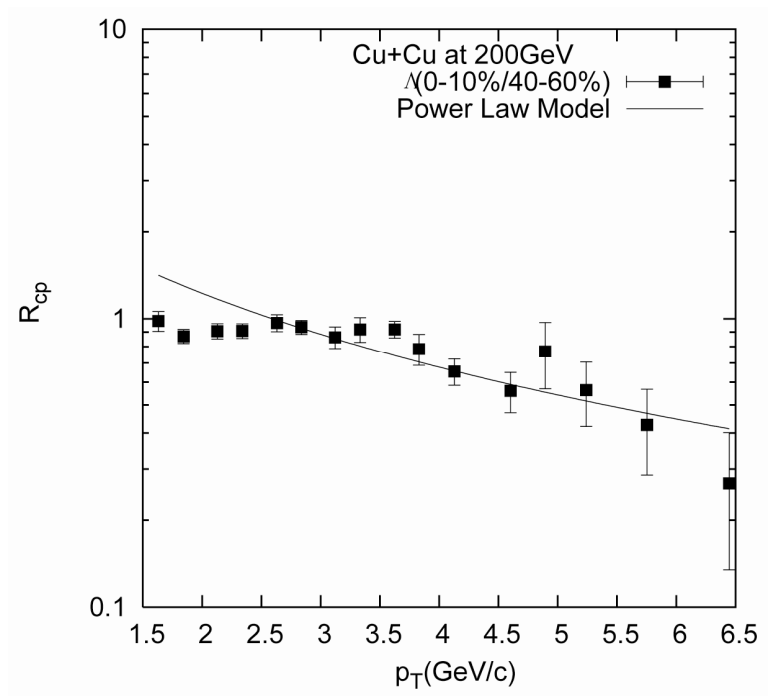

(b)

Figure 8. Plots of the nuclear modification factor $\left(R_{c p}\right)$ versus $p_{T}$ spectra for $\mathrm{Cu}$-Cu collisions at $\sqrt{s}=200 \mathrm{GeV}$. The experimental data are taken from Ref. [18]. The solid curves indicate the power-law-based description of the data.

Table 8. Calculated values of average transverse momentum $\left\langle p_{T}\right\rangle$ for $y_{c m}<0.5$ at $\sqrt{s_{N N}}=200 \mathrm{GeV}$.

\begin{tabular}{ccc}
\hline Nature of Collisions & Secondaries & Value of $\left\langle p_{T}\right\rangle$ in $\mathrm{GeV} / \mathrm{c}$ \\
\hline$k_{s}^{0}$ & 0.52 \\
p-p Collisions & $k^{+}$ & 0.45 \\
& $\Lambda$ & 0.48 \\
& $\bar{\Lambda}$ & 0.52 \\
& $\Xi^{-}$ & 0.50 \\
Au-Au Collisions & $\Xi^{+} b a r$ & 0.51 \\
& $k^{+}$ & 0.86 \\
$k^{-}$ & 0.68 \\
(Central) & $\Lambda$ & 0.50 \\
& $\Xi^{-}$ & 0.54 \\
$\Xi^{-}$ & 0.52 \\
& $\Xi^{+} b a r$ & 0.38 \\
$\Omega^{-}+\Omega^{+}$ & 0.28 \\
\hline
\end{tabular}

quite considerable. If these limitations are taken into account, the importance of this comprehensive work, though entirely phenomenological, assumes some degree of importance. Barring these generalized comments on an overall basis, we have some specific observations as well, which are also quite well-merited and are being presented hereafter: 1) The power indices for all the varieties of strange hadrons lie in the most cases,in the range of $10-12$. This is in accord with the prescription on the limit set by Brodsky [20], with the q-values bordering on the values, 2 - 3. 2) The $\chi^{2} /$ ndf values for production of cascade and omega particles have suffered quantitatively due to very small values of the number of degrees of freedom. 3) Thirdly, the $q$ and $n$ values do not exhibit any clear centrality-dependence or the mass-dependence of the observed heavy secondaries. 4) However, we cannot ascertain at this moment the properties of these parameters with regard to the nature of their energydependence(s), if any. 5) The average momentum values of these measured heavy strange baryons are found to be quantitatively compatible with other non-strange light hadrons, though the expression for the average transverse momentum is not very rigorously derived, for which reliability of expression (3) is certainly limited. 6) Some ratio-values shown by Figures 7 (a) and (b) are modestly well-described. 7) However, the values of the nuclear modification factor, denoted by $R_{C P}$, are not reproduced satisfactorily, especially on the lower-side of the $p_{T}$-values. But this is no wonder, as the used power laws are suited to high- $p_{T}$ values as was remarked above very concretely. 7) Still, with one of the simplest approaches, that we have succeeded in explaining the characteristics of a large bulk of data on these rare hadrons is certainly quite stimulating to and encouraging for us.

\section{Concluding Remarks}

Let us now sum up;

1) The used power laws explain quite well the mea- 
sured data on the observables like, $p_{T}$-spectra, some ratio-behaviours and the nuclear modification factors; so none of the questions related to suppression or enhancement is consequential. 2) The centrality-dependences of the $p_{T}$-spectra of strange hadrons too are well-reproduced. 3) The essential physical content of the power-law models is the modest observance of the $p_{T}$-scaling (as is reflected in the $\sim p_{T} / p_{0}$ term). And in terms of the functional efficacy, this model seems, so far, to be at par, if not better, with all other numerous existing approaches within the frameworks of either the Hadronic transport models or the statistical models [21-25]. 4) The model obviously bears no relationship with the concept of the quark-gluon plasma (QGP), which is, still, just a conjecture with so far no clear and concrete experimental support. 5) In a conclusive vein, this has to be asserted that we observe nothing too strange about "strangeness" production in high energy interactions.

The latter two points in the preceding paragraph highlight, in the main, the novelty of this study from a global viewpoint against the background of the current trends and streaming ideas in the present day Particle Physics.

\section{Acknowledgements}

The authors express their thankful gratitude to the honourable referee for making some valuable comments and suggestions.

\section{REFERENCES}

[1] H. Agakishiev, et al., "Strangeness Enhancement in $\mathrm{Cu}+$ $\mathrm{Cu}$ and $\mathrm{Au}+\mathrm{Au}$ Collisions at $\sqrt{s_{N N}}=200 \mathrm{GeV}$," $\mathrm{Nu}$ clear Experiment, arXiv:1107.2955v1, 2012.

[2] S. Schuchmann, "High $p_{T}$ Supression of Lamda and $k_{s}^{0}$ in Pb-Pb Collision at $\sqrt{s_{N N}}=2.76 \mathrm{TeV}$ with AL- ICE," Journal of Physics G: Nuclear and Particle Physics, Vol. 38, No. 12, 2011, p. 124080.

[3] P. Koch, B. Muller and J. Rafelski, "Strengeness in Relativistic Heavy Ion Collisions," Physics Reports, Vol. 142, No. 4, 1986, pp. 167-262.

[4] B. Muller, "Strangeness and the Quark-Gluon-Plasma: Thirty Years of Discovery," Invited Talk Presented at SQM 2011, Cracow, 18-24 September 2011.

[5] B. I. Abelev, et al., "Strange Particle Production in $\mathrm{p}+\mathrm{p}$ Collisions at $\sqrt{s_{N N}}=200 \mathrm{GeV}$," Physical Review $C$, Vol. 75, No. 6, 2007, Article No. 064901. doi:10.1103/PhysRevC.75.064901

[6] B. I. Abelev, et al., "Systematic Measurements of Identified Particle Spectra in pp, d + Au and $\mathrm{Au}+\mathrm{Au}$ Collisions at the STAR Detector," Physical Review C, Vol. 79, No. 3, 2009, Article No. 034909. doi:10.1103/PhysRevC.79.034909

[7] M. GaZ'dzicki and M. I. Gorenstien, "Power Law in
Hadron Production," Physics Letters B, Vol. 517, No. 3-4, 2001, pp. 250-254.

[8] P. Darriulat, "Large Transverse Momentum Hadronic Processes," Annual Review of Nuclear and Particle Science, Vol. 30, No. 1, 1980, pp. 159-210.

[9] S. Lehmann, A. D. Jackson and Benny ELautrup, "Measures and Mismeasures of Scientific Quality," Physics, arXiv:0512238v2, 2006.

[10] I. Sarcevic, "Proceedings of Large Hadron Collider Workshop," Vol. 2, Aachen, 1990, pp. 1214-1223.

[11] T. S. Biro and G. Purcsel, "Non Extensive Boltzmann Equation and Hadronization," Physical Review Letters, Vol. 95, No. 16, 2005, Article No. 162302. doi:10.1103/PhysRevLett.95.162302

[12] G. Arnison, et al., "Transverse Momentum Spectra for Charged Particles at the CERN Proton-Antiproton Collider," Physics Letters B, Vol. 118, No. 1-3, 1982, pp. 167-172.

[13] R. Hagedorn, "Multiplicities, $p_{T}$ Distributions and the Expected Hadron $\rightarrow$ Quark Gluon Transition," Revista Del Nuovo Cimento, Vol. 6, No. 10, 1983, pp. 1-50.

[14] T. Peitzmann, "Scaling Properties of Pion Production," Physics Letters B, Vol. 450, No. 1-3, 1999, pp. 7-14.

[15] C. Albajar, et al., "A Study of the General Characteristics of Proton-Antiprotoncollisions at $\sqrt{s_{N N}}=0.2-0.9 \mathrm{Tev}$," Nuclear Physics B, Vol. 335, No. 2, 1990, pp. 261-287.

[16] B. I. Abelev, et al., "Identified Baryon and Meson Distributions at Large Transverse Momenta from $\mathrm{Au}+\mathrm{Au}$ Collisions at $\sqrt{s_{N N}}=200 \mathrm{GeV}$," Physical Review Letters, Vol. 97, No. 15, 2006, Article No. 152301. doi:10.1103/PhysRevLett.97.152301

[17] M. Heinz, "How Well Does NLO pQCD Describe Strangeness Production in $\mathrm{p}+\mathrm{p}$ Collisions at $\sqrt{\mathrm{s}}=200 \mathrm{GeV}$ in STAR?" Journal of Physics G: Nuclear and Particle Physics, Vol. 31, No. 6, 2005, pp. S1011-S1014. doi:10.1088/0954-3899/31/6/048

[18] Y.-F. Wang, F.-L. Shao, J. Song, D.-M. Wei and Q.-B. Xie, "Centrality Dependence of $p_{T}$-Spectra for Identified Hadrons in $\mathrm{Au}+\mathrm{Au}$ and $\mathrm{Cu}+\mathrm{Cu}$ Collisions at $\sqrt{s_{N N}}=200 \mathrm{GeV}$," Chinese Physics C, Vol. 32, No. 12, 2008, pp. 976-983.

[19] J. Adams, et al., "Scaling Properties of Hyperon Production in $\mathrm{Au}+\mathrm{Au}$ Collisions at $\sqrt{s_{N N}}=200 \mathrm{GeV}$," Physical Review Letters, Vol. 98, No. 6, 2007, Article No. 062301. doi:10.1103/PhysRevLett.98.062301

[20] S. J. Brodsky, "Novel QCD Phenomena," Proceedings of Science LHC 07, Jyvaskyla, 23-27 March 2007.

[21] C. Blume and C. Markert, "Strange Hadron Production in Heavy Ion Collisions from SPS to RHIC," Particle and Nuclear Physics, Vol. 66, No. 4, 2011, pp. 834-879.

[22] J. Rafelski and J. Letessier, "Critical Hadronization Pressure," Journal of Physics G: Nuclear and Particle Physics, Vol. 36, No. 6, 2009, Article No. 064017. doi:10.1088/0954-3899/36/6/064017 
[23] W. Cassing and E. L. Bratkovskaya, "Hadronic and Electromagnetic Probe of Hot and Dense Nucleon Matter," Physics Reports, Vol. 308, No. 2-3, 1999, pp. 65-233.

[24] G. Torrieri, "My Strange Times with Johann Rafelski," Proceedings of SQM Conference Polish Academy of Arts and Sciences, Cracow, 2011.

[25] G. Torrieri, "Strangeness and Charm in Heavy Ion Collisions: Business as Usual or Phase Transition?" Proceedings of SQM Conference Polish Academy of Arts and Sciences, Cracow, 2011. 